\title{
Survey of Human Gait Analysis and Recognition for Medical and Forensic Applications
}

\author{
Shantanu Jana, Jadavpur University, India \\ Nibaran Das, Jadavpur University, India \\ Subhadip Basu, Jadavpur University, India \\ Mita Nasipuri, Jadavpur University, India
}

\begin{abstract}
Gait is a behavioural biometric which sometimes changes due to diseases, but it is still a strong identification metric that is widely used in forensic works, state biometric preserve sectors, and medical laboratories. Gait analysis sometimes helps to identify a person's present mental state which reflects on physiological therapy for improved biological system. There are various gait measurement forms which expand the research area from crime detection to medical enhancement. Many research works have been done so far for gait recognition. Many researchers focused on skeleton image of people to extract gait features, and many worked on stride length. Various sensors have been used to detect gait in various light forms. This paper is a brief survey of works on gait recognition collected from various sources of science and technology literature. The authors have discussed a few efficient models that worked best as well as a few of the data sets available.
\end{abstract}

\section{KEYWORDS}

Binary Coding, Gait Databases, Gait Recognition Survey, Kinect Skeleton Model, Morphological Skeleton, Silhouette Extraction

\section{INTRODUCTION}

Gait is ones walking pattern from which we can recognize a person. Gait recognition helps to recognize a person from behind, from far distance and in low light condition. C. D. Lim et al. (Lim et al., 2015a) Described in their paper that how gait analysis can improve gait of Parkinson Disease patient. In this work, they used depth camera attached with robotic walker to capture leg movement of patient. Parkinson Diseases effect on nervous system. In this disease, people lose their ability to move limbs in proper way and find difficulty in moving and walking. Symptoms continue and limbs become dysfunctional over time. Authors in this work, stored stride length and gait speed of few selected healthy older people whom they called control group and they selected few Parkinson Disease patient whom they called PD group. All people were male and aged between 69-92 years. They found that average

\section{DOI: 10.4018/IJDCF.289432}

This article published as an Open Access article distributed under the terms of the Creative Commons Attribution License (http://creativecommons.org/licenses/by/4.0/) which permits unrestricted use, distribution, and production in any medium, provided the author of the original work and original publication source are properly credited. 
stride length of control group is $37.2 \mathrm{~cm}$ which is greater than the stride length of PD group 22.38 $\mathrm{cm}$ but gait speed is almost equal in both cases. Early detection of Parkinson diseases (Kondragunta et al., 2019; Mallikarjuna et al.,2020) can delay the effect of this disease. For early detection of this disease deep learning algorithms can be used to estimate 2D poses. Then this $2 \mathrm{D}$ poses are projected in a 3D environment to extract gait parameters such as stride length, step angle. Deep learning is highly effective for $2 \mathrm{D}$ pose estimation. Mapping depth data with the $2 \mathrm{D}$ pose estimation produce $3 \mathrm{D}$ pose which helps to analysis gait for early detection. Rida et al. (Rida et al., 2015) showed that gait can be influenced by people clothing and carry bags which are termed as(Ali et al.,2011)covariates. They applied gait energy image to detect features. All feature vectors are processed with mask based variants. This work helps to identify gait in various clothing condition and was an improved system which elaborately explain ratio of the number of well classified samples over the total number of samples. Y. Qi et al. (Qi et al, 2016) describes gait phase detection from foot trajectory. They detected accurate gait cycle with error $-0.02 \pm 0.01$ second and stance phase with error $0.04 \pm 0.03$ second and swing phase with error $-0.05 \pm 0.03$ second. The aim of the work is to detect the influence of walking speed over gait spatio-temporal parameters. Gait analysis is widely used (Mastrigt et al., 2018) in forensic works. In several criminal trials in Europe, forensic gait analysis plays a major role in last 15 years. Clinical gait analysis and forensic gait analysis are not a completely different field of study because almost every feature is used in forensic gait analysis which are used in clinical gait analysis. These features include person's posture, movement of hands, head tilt, distribution of weight, angle of knee joint during walking, strides length etc. Gait recognition is widely used in forensic works for its discriminating ability from person to person (Muramatsu et al., 2013). In his $\mathrm{PhD}$ thesis (Seely, 2010) Richard D. Seely described large multi-biometric dataset that was captured as video processing and later it was reconstructed as a 3D dataset. Introduction of prototype biometric tunnel and its evaluation through rigorous analysis of dataset and sensor modification gives satisfactory result to identify discriminating boundary of gait recognition. Gait recognition can be done (Sun et al.,2019) using deep learning to find the key points of skeleton from image. This helps to recognise weather the subject is walking or running. The key points of human skeleton are very important for human behaviour recognition and also posture detection. Event based (Wang et al., 2019) gait recognition, EV-Gait shows $96 \%$ recognition accuracy on DVS128 and EV-CASIA-B gait databases. It is considered that dynamic vision sensors (DVS) are new sensing modality for object recognition. It generates asynchronous noisy events. EV-Gait is able to work with this noisy event streams and showed a high accuracy level in gait recognition. Gait recognition (Sokolova andKonushin,2019) by neural features obtained through DVS has also shown high accuracy rate which is more than $98 \%$ for TUM-GAID gait data set. Cross-view gait (Chao et al.,2019) recognition form Gait Set shown 95.0\% average rank-1 accuracy on CASIA-B dataset without covariates and this accuracy rate is $87.2 \%$ with bag-carrying and $70.4 \%$ with coat-wearing on CASIA-B data set. According to the psychological study (Boyd and Little, 2005) gait has small but very strong features for recognition of individual gait. There are several factors that can confuse the gait recognition system. These factors include variation of footwear, injury, change of age. Quasi gait recognition is very effective for gait recognition because it is less sensitive to these factors. Independent component analysis ICA (Lu and Zhang, 2007) and modified ICA (Rani and Arumugam, 2010) are also very effective for gait recognition. ICA is similar like Principal component analysis PCA where the difference is that ICA has statistically independent components. In research work on gait and posture (Papi et al., 2018) it is found that peak knee sagittal angels are plays an important role to diagnose movement disorder. Diseases like ataxia, restless legs syndrome, Parkinson's disease, and stroke are common sign movement disorder. In these cases, knee angles are interest of study of bio clinical laboratories. Recent development of wearable technologies (Margiotta et al., 2016; Papi et al., 2017; Duc et al., 2014) has made such measurement easy and accurate. Rehabilitation of stroke patients who have survived from trauma (Carbonaro et 
al., 2015) needs a daily life monitoring system. A textile goniometer work on knitted piezoresistive fabrics (KPF) developed to measure knee flexo-extension activities. This work (Chao et al., 2019) compares the performance of textile goniometer with inertial measurement unites(IMU). IMUs are the gold standard wearable motion sensors widely used. Goniometer gives same reading information of IMUs. This new light weighted goniometer gives extra set of information which IMUs does not. Ataxia (Putzhammer et al.,2005), schizophrenia are such diseases in which people lose his ability to think, feel, behave properly, has common motor disabling symptoms. Gait analysis for schizophrenia patient produces day to day mental ability improvement data for medical analysis. Anatomy of human nervous system (Sanders and Gillig,2010) reveals that walking messages are controlled by motor, premotor cortex, subcortical nuclei, brainstem and cerebellum. Motor and premotor cortex generates initial signal for walking and others are responsible for modifying the signal. Gait reflects every kind of nervous system condition. This paper gives a cause of different abnormal gait for different diseases and drug effect. Asymmetry stride length disturbed due to short in cortical and basal ganglia disorders. Chronic alcoholic patient and drug effect cause ataxic gait abnormality. Many works had been carried out on gait analysis for medical treatment.

\section{GAIT DATABASES}

A number of gait databases were developed by different research groups working in the area of gait recognition. Among them Silhouettes gait database is most basic gait database. These gait databases are captured through different sensors and in different light conditions. CASIA gait database, NLPR gait database, CMU Mobo database, MIT, USH, UMD are all rich gait databases. Detailed discussion of each of these databases is given below. Table I. shows different gait database description.

\subsection{Silhouette Gait Database}

Silhouettes description of gait images are captured in specific light condition with darker backgrounds. Silhouette image represent shape of object in one colour. Various silhouette extraction methods (Schiel et al., 2013; Lu et al., 2007; Zhuang and Chen, 2007) are introduced to extract gait images from that. CASIA, NLPR have good collection of silhouette database. Some sample images from NLPR database is shown in Figure 1.

Figure 1. Silhouette gait images from NLPR gait database (Martek, 2010)
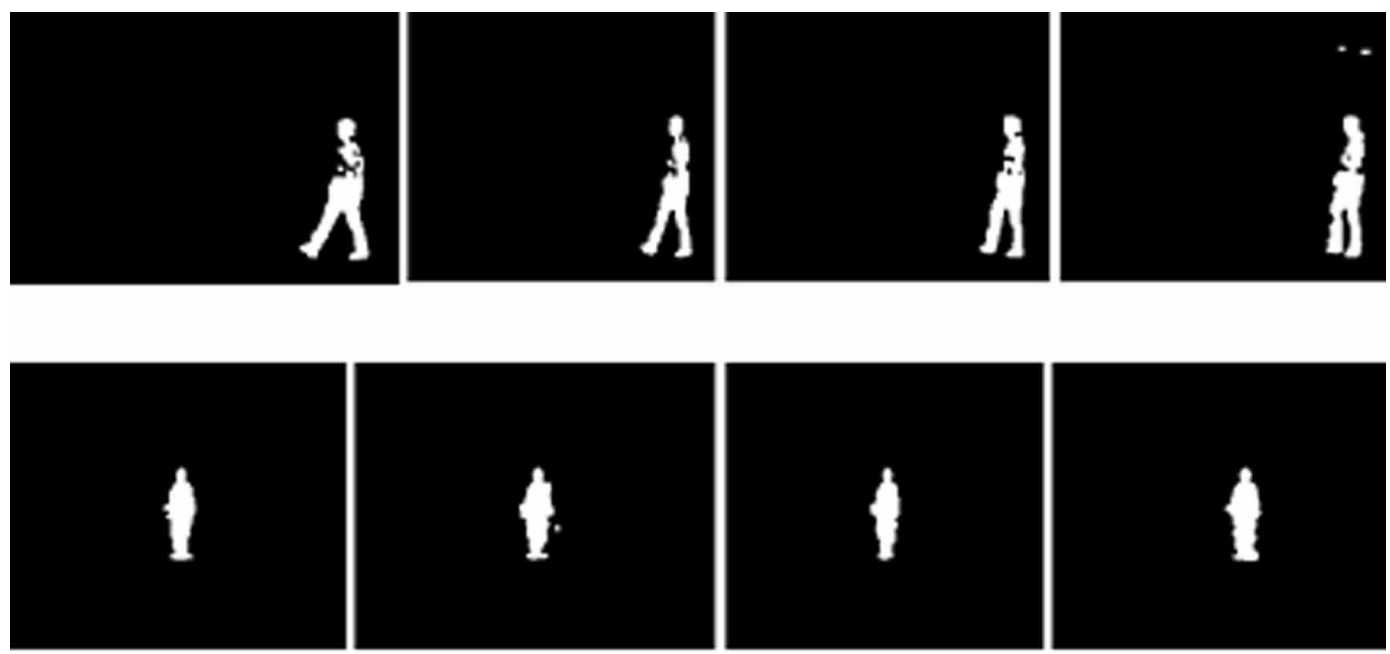

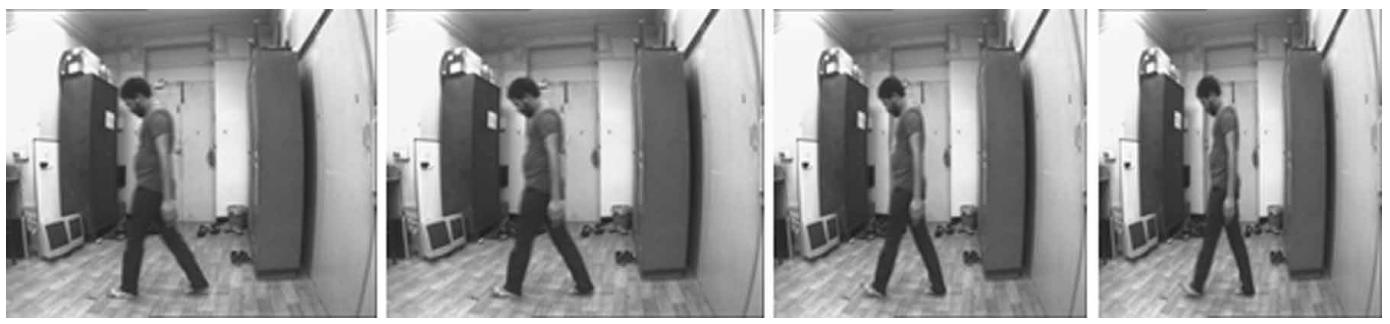

\subsection{Near Infrared Gait Database}

NIR imaging is capable to capture images in low light condition. It is more effective than a grey scale converted image. One can mount (Li et al., 2006; Firmenichy et al., 2011) NIR light emitting diodes and use various filters to get desired NIR images. Active NIR illumination (Firmenichy et al., 2011) produces invisible marker tracking, stereo depth estimation. Images which are taken in nonvisible spectrum gives NIR imaging an added advantage over RGB imaging. Choosing particular bands over the range of NIR spectra (from 700 to 1100 nanometres) create multispectral NIR image. Some sample NIR images are shown in Figure 2 taken at 416 lux and walking distance was 3 meter.

\subsection{Thermal Gait Database}

Thermal imaging is most effective to detect covariates in gait recognition (Kolaric et al., 2011;Hikal,2013;Razeghi et al.,2007). Every object emits electromagnetic radiation. This electromagnetic radiation is thermal energy that is invisible to human eyes. Various thermal cameras have such sensors to capture this heat energy and such imaging is called thermography. The images thermal camera captures are thermo grams. Infrared spectral range of thermal imaging is between 9,000-14,000 nanometres. Figure 3 are thermal images collected at CMATER laboratory, Jadavpur University. These images ware taken at maximum lux 89 and minimum lux 11. Walking distance ware 10 meter.

\subsection{Multiview Gait Database}

Walking styles are captured from various viewpoints to gain maximum gait features which increase recognition success rate. Multiview (Figure 4) gait databases are prepared to get angular motion of object in respect of gait features with multiple cameras installed in various point or a single camera in a fixed position.

Figure 3. Thermal gait images(Razeghi et al.,2007)
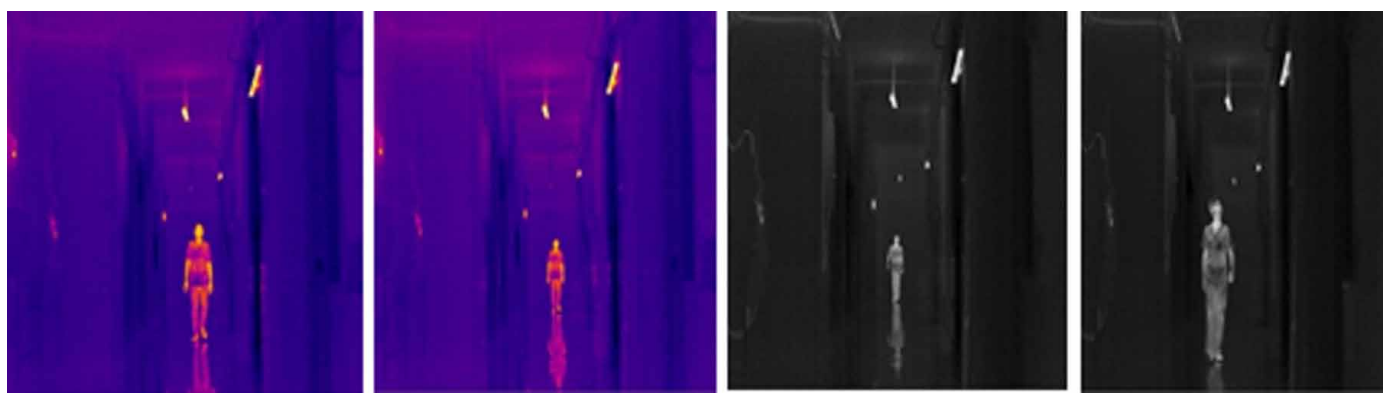

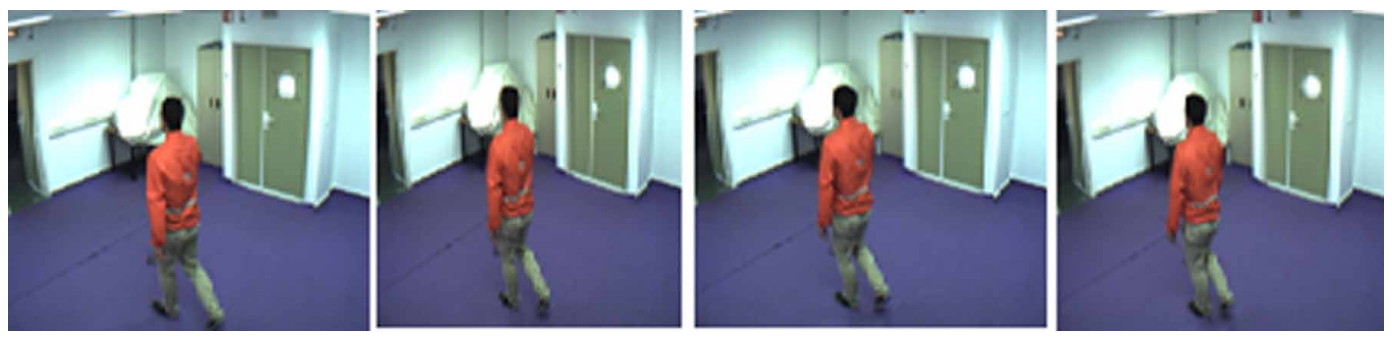

\subsection{Large Sequence-Based Human Gait Database}

This type of imaging needs protocol oriented camera installation. Basically two types of views needed to capture large sequence of gait images. Normal and oblique installation can be put in multiple positions (Shutler et al., 2002) to construct large sequence based imaging (Figure 5). SOTON HiD is a good collection of large sequence based gait database. This database was prepared by University of Southampton. This database consists of 100 subjects each having 8 sequences captured at 25 frame per second.

\section{DIFFERENT FEATURE EXTRACTION METHODOLOGIES}

Feature selection is state of the art of the entire recognition system development process which expects ground truth from various modes of imaging system. From early history of gait recognition many databases were prepared and various methods of feature extraction were developed. Overall few feature extraction techniques are key sources of further development of advance functions.

Figure 6. Shows a generalised version of gait recognition system. All the components of the system are described briefly and results are given for few individual components as training and testing feature vector.

Figure 5. Camera installation for large sequence base gait database (Shutler et al., 2002)

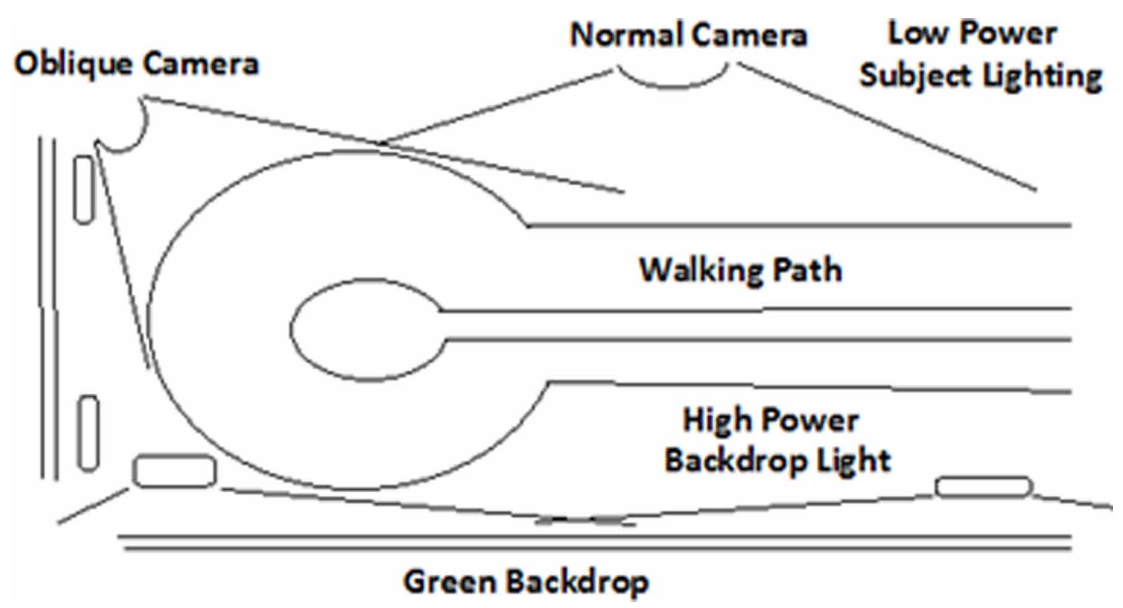


Table 1. Different gait database description

\begin{tabular}{|c|c|c|c|}
\hline Database name & Database type & Number of subjects & Organization \\
\hline $\begin{array}{l}\text { CASIA Dataset B (Yu et } \\
\text { al.,2006) }\end{array}$ & Multi View & 124 & $\begin{array}{l}\text { Institute of Automation of } \\
\text { Chinese Academy of Sciences }\end{array}$ \\
\hline $\begin{array}{l}\text { CASIA Dataset C (Arai and } \\
\text { Asmara et al.,2012) }\end{array}$ & Infrared & 153 & $\begin{array}{l}\text { Institute of Automation of } \\
\text { Chinese Academy of Sciences }\end{array}$ \\
\hline $\begin{array}{l}\text { CASIA Dataset D (Zheng et } \\
\text { al.,2012) }\end{array}$ & camera and Rscan Footscan & 88 & $\begin{array}{l}\text { Institute of Automation of } \\
\text { Chinese Academy of Sciences }\end{array}$ \\
\hline NLPR (Wang et al.,2003) & Silhouette & 20 & $\begin{array}{c}\text { National Laboratory of Pattern } \\
\text { Recognition }\end{array}$ \\
\hline $\begin{array}{c}\text { CMU Mobo (Sivapalan et } \\
\text { al.,2011) }\end{array}$ & $\begin{array}{l}\text { Six high resolution colour } \\
\text { camera and treadmill }\end{array}$ & 25 & $\begin{array}{l}\text { Robotics Institute, Carnegie } \\
\text { Mellon University }\end{array}$ \\
\hline MITAI (Cuntoor et al.,2003) & Raw image sequence data & 24 & $\begin{array}{c}\text { Massachusetts Institute of } \\
\text { Technology }\end{array}$ \\
\hline $\begin{array}{l}\text { GMU gait database (Wen et } \\
\text { al.,2010) }\end{array}$ & Large sequence-based & 16 & George Mason University \\
\hline $\begin{array}{c}\text { HID-UMD (Cheng et } \\
\text { al.,2008) }\end{array}$ & Multi view & 55 & University of Maryland \\
\hline $\begin{array}{l}\text { AVAMVG (Fernandez et } \\
\text { al.,2014) }\end{array}$ & Multi view & 20 & University of Cordoba \\
\hline $\begin{array}{c}\text { SOTON HiD (Shutler et } \\
\text { al.,2002) }\end{array}$ & Large sequence-based & 115 & University of Southampton \\
\hline
\end{tabular}

Figure 6. Block diagram of generalised system of gait recognition (Pushpa et al., 2010)

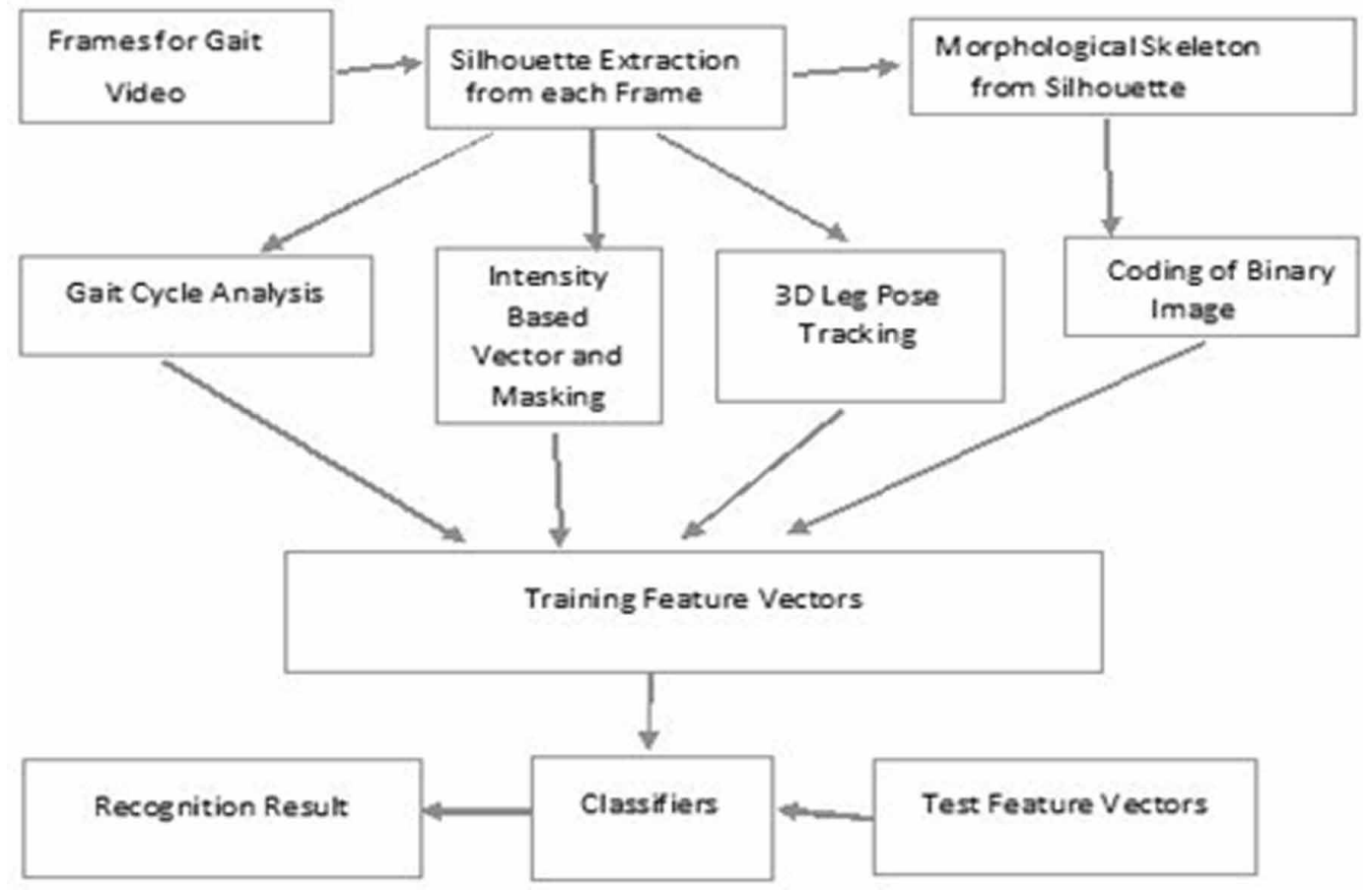




\subsection{Silhouette Extraction}

Silhouette Extraction methods employ background elimination. Several background elimination techniques ware proposed in various research works. Many works have been done on video processing where a fixed back ground is not possible for image subtraction due to illumination change of light sources. Sometimes movable cameras are installed in object location to capture forensic data which need advanced background elimination technique. Outdoor environments where light source intensity is time dependent also need these types of background elimination models. Few established background elimination techniques include Horprasert's Algorithm (Ahn and Byun, 2007) Region Based Background Subtraction, (Kim et al., 2007; Babacan and Pappas,2007) Laplacian Distribution (Jan and Wang,2012) Gaussian Distribution (Panda and Meher,2013). Horprasert's Algorithm (Gomez et al., 2012) works on batch mode to separate the brightness of its chromaticity components. This algorithm uses statistical background model. A region based background subtraction divide image in region pixel. It first draws a minimum bounding box around the desired subject then transform the image by mean shift segmentation. Pixel object likelihood and naïve region likelihood find foreground image from segment image. Laplacian distribution identifies the intensity change rate on the image boundary to compare with the other pixel region. Gaussian distribution analysis all pixel intensity in exponential domain.

\subsubsection{Gaussian Distribution}

Gaussian distribution represents bell shaped curve in terms of exponential function. It identifies specific area of interest in an image from standard normal $\mathrm{z}$ table:

$$
f(x)=\frac{1}{\sqrt{2 \pi \sigma^{2}}} e^{\frac{-(x-\mu)^{2}}{2 \sigma^{2}}}
$$

$f(x)$ Represents a Gaussian function in equation (1). In this equation, ' $x$ ' represents each pixel value where ' $\mu$ ' and ' $\sigma$ ' represent mean and standard deviation respectively. E. Juana et al. (Juana et al., 2014) Introduced a background subtraction model to complex environments. They proposed that their model can improve any Gaussian based models to detect complex back ground.

\subsubsection{Mixture of Gaussians}

Gaussian Mixture model is the mixture of weighted sum of Gaussian distribution components. It is described in paper by (Zhao and Du,2010) Stauffer and Grimson that if a pixel z(x y) is chosen from a video sequence at any time $t$ as represented in time series of video sequence then it represent its history at that particular time. The history of the pixel in video sequence is $z^{1}$ to $z^{t}$ which can be observed by a mixture of $\mathrm{K}$ Gaussian distribution. The probability of observing the pixel at particular time $t$ can be represented by following equation:

$$
\mathbf{P}(\mathbf{z t})=\sum_{i=1}^{K} w_{i, t} \times f\left(z_{t}(x, y), \mu_{i, t}, C O V_{i, t}(x, y)\right)
$$

In equation (2), $\mathrm{w}_{\mathrm{i}, \mathrm{t}}$ is the estimated weight of $\mathrm{i}_{\mathrm{th}}$ Gaussian in the mixture at time $\mathrm{t} . \mu_{\mathrm{i}, \mathrm{t}}$ Is mean and $C O V_{t}(x, y)$ is the covariance of the $\mathrm{i}_{\text {th }}$ Gaussian at time $t$. Gaussian probability density function is represented in equation (3). Equation (4) represent the covariance matrix form. Every new pixel $\mathrm{Z}_{\mathrm{t}}$ is examined with all $\mathrm{K}$ Gaussian distributions until a match found. They call it a match if it is 
within 2.5 standard deviation of a distribution. In equation (3), ' $n$ ' is tuning variable that stabilize system to desired configuration:

$$
\begin{aligned}
& f\left(z_{t}(x, y), \mu_{i, t}, C O V_{i, t}(x, y)\right)=\frac{1}{\sqrt{2 \pi^{n} \times\left|C O V_{i, t}(x, y)\right|}} e^{-\frac{\left(z_{t}(x, y)-\mu_{i, t}\right)^{T}}{2 \sum_{1}^{k}\left(z_{t}(x, y)-\mu_{i, t}\right)}} \\
& C O V_{k, t}(x, y)=\left\{\sigma_{k}^{2} x: x=y\right\}
\end{aligned}
$$

If no pixel matches with any $\mathrm{k}$ distribution, then least probable distribution is replaced with mean. The prior weight of ' $k$ ' distribution at time' $t$ ' is represented as by the following equation:

$$
w_{k, t}=(1-\alpha) w_{k, t-1}+\alpha\left(M_{k, t}\right)
$$

where $\alpha$ is square of the learning rate. If model match then variable $M_{k, t}$ set to 1 and 0 for other. $\frac{1}{\alpha}$ determines the derivative of distribution parameters. $w_{k, t}$ is the causal low pass filter and if model matches then new update function can be produced to update $w$ and $\alpha$ shown in paper evaluated by P. KaewTraKulPong et.al. (Kaewtrakulpong and Bowden, 2001).

\subsubsection{Background Estimation}

When the object in scene moves, its variance become larger than background pixel. It can be identify which of the Gaussian produced by back ground. If objects are not fixed in the scene, then the Gaussians are not matched with exiting Gaussians. In this case new distribution has to be produced or the variance of the existing distribution has to be increased. In a static fixed object scene first measure $\frac{w}{\sigma}$. This value increases when the distribution gain more evidence and variance decreases. Gaussian distribution should be searched heuristically to find most supporting evidence and least variances which is the background. If variances are order in a list, then equation is as follows:

$$
B=\arg \min _{b} \sum_{k=1}^{b} w_{k}>T
$$

In equation (6), the first $\mathrm{B}$ distributions are model as background and $\mathrm{T}$ is the measure of how much data is accumulated from portion of background. Foreground pixel is any pixel that differs by 2.5 standard deviations than B distribution.

\subsubsection{Advanced Changes for Background Subtraction}

E. Juana et al. (Juana et al,2014) proposed an advance algorithm to background subtraction for complex environment. They modified variance $\left(\sigma^{2}\right)$ and learning rate $(\alpha)$ of Mixture of Gaussian. They introduced new distance matric. They used spatio-temporal region representation instead of pixel based representation. 
Background pixel can be found in each colour channel and assign a minimum variance value for each colour component. Consideration of colour channel for pixel classification reduces noise that adds at the time of segmentation. New algorithm to modify variance says in following order:

$$
\begin{aligned}
& \forall z \in(1 \rightarrow n) \\
& \text { If } \sigma_{k, z}<\min \left(\sigma_{z}\right) \\
& \sigma_{k, z}=\min \left(\sigma_{z}\right)
\end{aligned}
$$

where $\mathrm{k}$ is the number of distribution and $\mathrm{n}$ is the number of channel in the colour space. The learning parameter $\alpha$ is a decreasing quasi global space-time variable. It filters illumination and decreases gradually to its min value as the system goes to stable condition. The definition of $\alpha(t)$ is as follows:

$$
\left.\alpha(t)=\min \left\{\alpha: \alpha(t)<\frac{1}{1+\log t^{m}}\right\}\right\}
$$

$\alpha(t)$ is learning rate which stabilizes converging procedure more accurate. Variable ' $\mathrm{m}$ ' is tuning parameter which is chosen as per desired configuration. A static scene with a static object has only one time varying learning rate where a scene with multiple moving objects have many different learning rates. The new definition of learning rate can deal with drastic change in illumination and reduce noise created by it. Instead of using pixel based representation, if spatio temporal representation is used then the new model can filter out high frequency motions. In this representation, three variables can be used to keep spatial and time information. Consider $\mathrm{x}$ and $\mathrm{y}$ keep spatial information and $\mathrm{t}$ keeps the time record then xyt forms cube of pixel information where $\mathrm{x}$, $\mathrm{y}$ and $\mathrm{t}$ represented by arithmetic mean. A distance matric ' $M$ ' that tells which Mixture of Gaussian is better to represent the current pixel is stated as follow:

$$
M_{k, j}=\frac{E\left[\left(x_{j}-\mu_{k, j}\right)\left(y_{j}-\mu_{k, j}\right)\right]}{\sigma_{k, j}}
$$

where ' $k$ ' represents distribution of mixture and ' $j$ ' represents color components YUV. This entire proposal of subtraction then implemented in to cascade algorithm described by 'L'. F.Teixeira et al. (Teixeira et al, 2007). Independent threshold for each color component YUV helps to reduce drastic illumination changes.

\subsection{Morphological Skeleton}

Morphological skeleton holds all the property of shape and size of the main object. Erosion and filling thinner the image to produce skeleton where dilation thicker the image preserving all property of shape and size of the image. Closing is property that fill the inside image areas. Erosion and dilation can be defined as follows:

$$
A \ominus B=\{(x, y)-(u, v):(x, y) \in A,(u, v) \in B\}
$$




$$
A \oplus B=\{(x, y)-(u, v):(x, y) \in A,(u, v) \in B\}
$$

This is simply the erosion and dilation of A by B. B is a structuring element that is a part of Minkowski set operation as input. The set operator's intersection, union, inclusion and complement then can be applied to image to produce different image behaviour. A. Maragos et al. (Maragos and Schafer, 1986) described that morphological transformations are upper semi continuous transformations if every structuring element is represented by a compact subset of $E$ where E represents Euclidian space. Some properties of erosion and dilation is as follows:

$$
A_{z} B=(A B)_{z}
$$

where $\mathrm{Z}$ is discrete space:

$$
A \ominus B=\{(x, y)-(v):(x, y) \in A,(v) \in B\}
$$

Equation (12) is a form of erosion which is a translation respect to a single point.

\subsubsection{Morphological Skeleton Property}

A skeleton subset is created by placing disks at particular position of the image object. This particular position is an arbitrary position on image object. Then apply set operation to get skeleton subset. The two set operations erosion and opening are performed to get skeleton subset. Then union of all such skeleton subset creates skeleton of the continuous image object X. Discs or squares are the structuring elements. They are isotropic in nature. Disc of radius ' $\mathrm{r}$ ' can be defined as $D_{r}$ :

$$
F_{n}=X \ominus D_{r}
$$

$$
\text { Fskel }=\bigcup_{n>0} F_{n}
$$

where $F_{n}$ is the $\mathrm{n}$ th image subset and Fskel is the skeleton of the image $\mathrm{X}$. This property is a power full tool to develop more efficient algorithms for skeleton construction. R.Kresch et al. (Kresch and Malah, 1998) described Lantu 'ejoul's formula as follows:

$$
F_{n}=X \ominus D_{r}-\left[\left(X \ominus D_{r}\right) \psi D_{d r}\right]
$$

where $\psi$ is the opening and $D_{d r}$ is a closed disc with infinitely small radius $d r$.

\subsubsection{Coding of Binary Image}

The most important coding techniques for binary images are block coding, run length coding, Elias coding. These three coding can be there after apply on skeleton subset. Skeleton subset is a binary image. Each skeleton subset can be divided in to blocks. Breaking into blocks make the computation faster but experiments show that instead of applying on subsets if binary coding can be applied on 
whole skeleton then it performs better. Applying coding on skeleton creates skeleton function. To code skeleton function $F_{F s k e l}(x)$, first code 'Fskel' form equation (15) by any of the three coding scheme said before. Then code grey tone information by Huffman code. Experiments say that Elias coding is best coding scheme to code a binary image than block Huffman or run-length-Huffman coding and Elias coding which gives best compression of original image. Table II Shows the efficiency of different coding scheme tested on $64 \times 64$ image pixel. $P=2 \times 4$ refers $2 \mathrm{~d}$ blocks each size is 8 pixels. Common indicates same Huffman code for black and white run lengths where as separate indicates different Huffman code. ' $m$ ' Represent the system in which the run length is represented $. m=3$ Means the representation in ternary system.

\subsection{Kinect Skeleton Model}

There are vast application of Kinect technology (Lun and Zhao, 2015) in healthcare. Medical experts need extensive analysis of physical therapy, rehabilitation training, and body movement based exercise of patients. Kinect technology gives them computer based detail analysis in affordable, convenient and user friendly way. Microsoft introduced Kinect technology as a low-cost motion sensing device. It provides 2D colour image frames, 3d depth image frames and 3D skeleton frames. Kinect SDK is combined with a model that represent full human skeleton. This model is trained with huge amount of labelled data. This trained model jointly with Kinect SDK gives real time skeleton tracking. Kinect skeleton model captures joints of human body by depth image analysis. Kinect is a senor that has a camera and a depth sensor to capture 3D movement of body and detect body joints. E. Gianaria et al. (Fritz and Lusardi, 2009) described that a Kinect sensor of Microsoft is able to detect 20 body joint in real time and in 3D space. Kinect provides gaming environment that can control a game by your body, gesture and voice. Using Kinect sensors to detect body joints help to study gait movements more accurately and those help researchers to develop more strong algorithms in this field.K.Arai et al. (Arai and Asmara,2013) worked on both, model free and model based features for gait recognition. Model free approaches are shape based features that are extracted from silhouettes and model based approach gathers features from skeleton parameters like legs, arms, neck. Knee angles can be extracted as a feature from model based approach. A.Sinha et al. (Sinha et al., 2013) used features like areas of upper and lower body parts and distances between the upper body centroid and the centroids derived from different joints of body. They calculated all the feature within half gait cycle. The experiment results are shown in table III. Features ' $x$ ' are collected from research work by A.Ball et al. (Ball et al.,2012) and ' $y$ ' features include area and distance features (Sinha et al.,2013). Total 32 features include 18 ' $x$ ' features and 14 'y' features. ' $x$ ' Features are lower limb joints angle.

\subsection{Intensity Based Vector and Masking}

GEI is Gait energy image which reveals many characteristics of silhouette gait image (Bharadwaj and Chanda,2020). The high intensity pixels denote static part of the body where low intensity pixels denote dynamic part of the body. This concept of feature extraction is more evident when pictures are captured in low frame rate condition from a moving camera:

$$
\begin{aligned}
& \left\{\left(x_{0}, y_{0}\right), \ldots,\left(x_{n}, y_{n}\right)\right\} \in I \\
& \mathrm{G}=\left\{I_{0}+I_{1}+\ldots I_{k}: I \in F_{t}\right\} \\
& G E=\frac{1}{k} \times G
\end{aligned}
$$


In the above mathematical derivation, ' $F$ ' is set of frames and ' $k$ ' is number of frames in time interval ' $t$ '. ' $I$ ' containing matrixes of image pixel where ' $\mathrm{x}$ ' and ' $\mathrm{y}$ ' is pixel coordinates. $G$ is sum of all such images within time interval ' $t$ '. Varying this ' $t$ ' and ' $k$ ' parameters one can create appropriate silhouette for such energy image analysis. Then (Kondragunta et al,2019; Yang et al,2014;Bashir et al,2008; Yang et al,2008) each row of the GEI can be taken as feature unit. Then compute sum of each row GEI intensity normalized by sum of all row intensity. Then this normalized intensity of each row creates feature vector for further processing. Then apply a mask to find the most significant feature vectors. After that apply a final mask to compute masked GEIM. The final mask (FMASK) is 'and' operation between the low intensity vectors, intensity vectors with carrying bag, intensity vectors with coat. Then following equation computes masked GEI:

$$
G E I M=G E I \times F M A S K
$$

\subsection{Gait Cycle Analysis}

Gait cycle can be divided into five different phases which are Initial Contact(IC), Load Response(LR), Mid Stance(MST), Terminal Stance(TST), Pre Swing Double Support(PSW) shown in figure 7. C. MacDonald et al. (MacDonald et al, 2007) explained three more gait phases as Initial Swing (ISW), Mid Swing $(M S W)$, and Terminal Swing (TSW).

Hip, knee, ankle angles and time can be considered as input parameters within these phases. Then a fuzzy system can be applied to the variables. Three membership functions are small(S), Medium (M), Large (L) which represent the output of the system. Results shown in table IV. Wearable motion detector is a sensor device that captures gait cycle in real-time. C.-C. Yang et al. (Yang et al., 2011) worked on five PD patients to study gait behaviour. A wearable motion detector is a tri-axial accelerometer that record acceleration during walking. Accelerometer device measure static and dynamic acceleration using earth gravity. Then record acceleration along Vertical, Antro Posterior, and Medio Lateral direction to analysis different gait movement. A pressure sensor used to measure foot pressure. This sensor contains a force-sensitive resister (FSR). This sensor detects ground reaction force and gait phase. If the sum of signals from FSR tends to zero then the phase is swing (Perikesit et al., 2011). An autocorrelation coefficients are then computed from signal points of each directional signal. Then measure most impotent coefficients for further processing from a series of autocorrelation coefficients. These coefficients then consider as feature vectors (Gafurov and Snekkenes, 2009). The first and last few second of walk are not natural gait of person. Average walking speed is gait cycle per second of a person. This speed changes person to person. Even for a particular person this speed sometimes varies in consecutive cycles. For this reason, a continuous signal captured from radar can be used to identify Doppler signature of the gait. Frequency transform and then extracting features from a five meter walk or identifying Doppler signatures are all eliminate periodical dissymmetry in gait. Step length (Sayeed et al., 2013; Rampp et al.,2015; Wen et al.,2013)

Figure 7. Different phases in gait cycle (MacDonald et al, 2007)

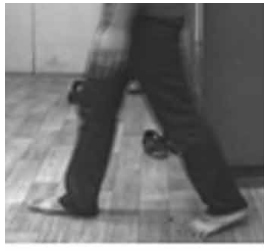

Initia1 Contact

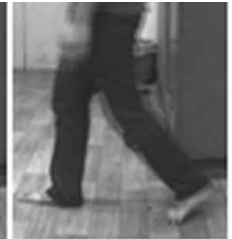

Loading Response

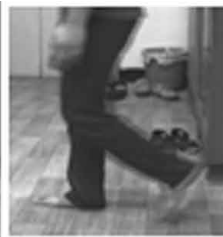

MidStance

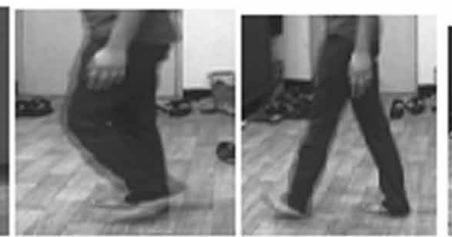

MidStance
Tem inal Stance

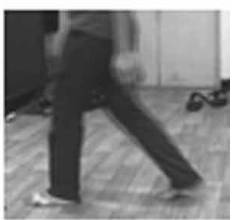

Pre swing Double Suppart 
is another important feature to recognize human gait. The most popular method to detect average step length is inverted pendulum model. In this model, measure the leg length from pelvis to hill, displacement of centre of mass in each step:

$$
\frac{d^{2} \theta}{d t^{2}}+\frac{h}{S L} \sin \theta=0
$$

In equation (20) SL is stride length, h is vertical displacement of centre of mass and $\theta$ is angular displacement. h can be found from above equation. Then average step length $(s l)$ can be computed by computing following equation:

$$
s l=\frac{1}{N} \sum_{n=0}^{N-1} 2 \times k \sqrt{2 h l-l^{2}}
$$

In equation (21), $\mathrm{N}$ is number of step lengths in one example. $\mathrm{K}$ is a calibrating constant which is a ratio between mean real and mean probable step length.

\subsection{D Leg Pose Tracking}

Gait analysis with 3D data explore more discriminative features. Motion capture (Mocap) data available are free database which gives 3D markers in major human joints. 3D pose data can be extracted form video data by using Annealed particle filter (Livne et al. 2012; Chang and Lin, 2010; Cho et al.,2013). Particle filter algorithm works on Bayesian framework for motion capture. The sampling process of particle filter associates with several weights that generates a single local maximum point that detects by particle filter. The importance of weight must be a probability $w=p\left(x_{n} \mid y_{n}\right)$. Where $x_{n}$ is the hidden state vector and $y_{n}$ is the observation. From this 3D model, first segment the human leg then identify the ankle joint. From this do 3D leg pose tracking and then do gait analysis from that. In 3D model consider each leg segment as cylinder. C. D. Limet al. (Lim et al., 2015b) identified leg posture from robotic walker. Skeleton of leg segments (Figure 8) can be identified from there. Then curvature of the leg segments can be found out which denotes the ankle

Figure 8. Skeleton construction of leg segment (Lim et al.,2015b)

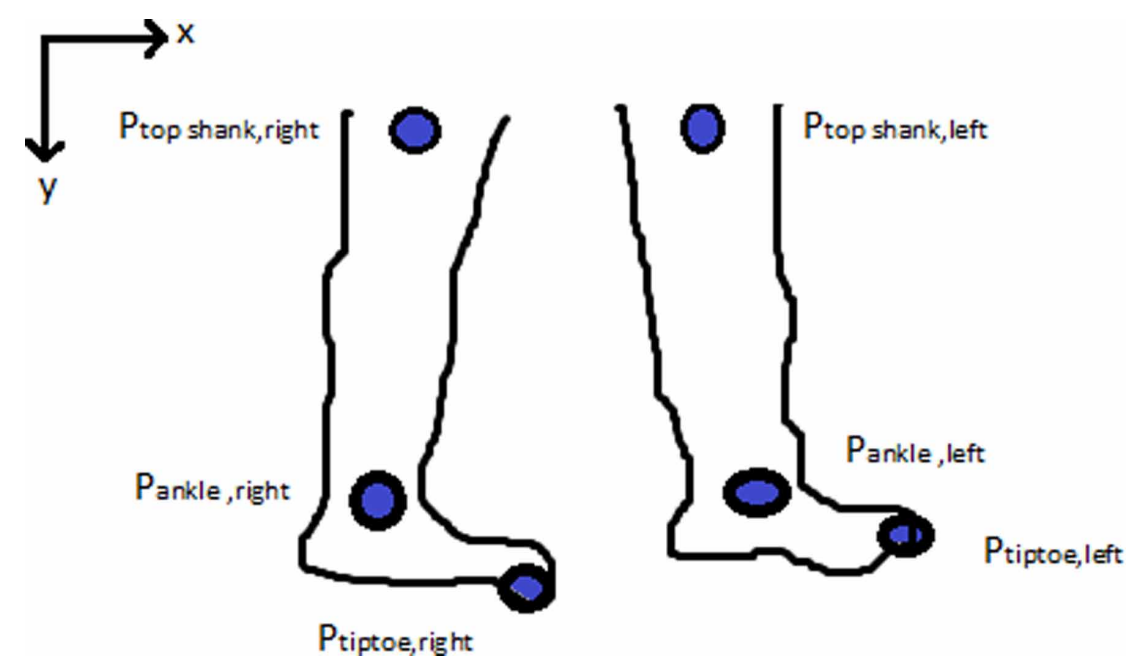


joint. To find out the curvature, do convolution between $s_{i-1}, s_{i}, s_{i+1}$. Where $s_{i}$ is the skeleton points. Then apply a second derivative mask to find transpose of the convolution points. Least negative curvature of skeleton depth values are position of ankle $p_{\text {ankel }}$. The two more position shank and toe are identified.

A state model $\underset{x}{\rightarrow}$ then computed to represent all the cylinders of leg segments. $\underset{x}{\rightarrow}$ contains 12 elements:

$$
X_{t}=\sum_{i=1}^{N} s_{i} w_{i}
$$

$s_{i}$ is the ith particle that represent leg pose and $w_{i}$ is the associated weight with it. $X_{t}$ is the state model for particular time ' $t$ '. The initial sampling of particle ' $s_{1}$ ' includes position of ankle, shank, toe and widths of ankle. To calculate weight vectors, first identify the contour of the leg segment. From this contour, generate one mean square error function $f_{1}\left(x_{n}, y_{n}^{1}\right)$. Where $y_{n}^{1}$ is observation of 'edge map' and $x_{n}$ is the sampled cylindrical model. Another mean square error function $f_{2}\left(x_{n}, y_{n}^{2}\right)$ generates from set of fore ground points $y_{n}^{2}$ and sampled cylindrical model $x_{n}$. Then an exponential function constructed by these two mean square error function shown in equation (23). Then, finally compute the weights calculating probability shown in equation (24):

$$
\begin{aligned}
& f_{\exp }\left(x_{n}, y_{n}\right)=\exp -\left(f_{1}\left(x_{n}, y_{n}^{1}\right)+f_{2}\left(x_{n}, y_{n}^{2}\right)\right) \\
& p\left(x_{n} \mid y_{n}\right)=\frac{1}{n} \sum_{1}^{n} f_{\exp }\left(x_{n}, y_{n}\right)
\end{aligned}
$$

Equation (24) shows particle weight calculated in particular time ' $t$ '. Table V shows comparison of step length with particle filter and without particle filter.

\section{RESULTS AND DISCUSSION}

Two different scheme of skeleton extraction and their results from previous work have shown in table 2 and 3. The first one is for morphological skeleton. Morphological skeleton creates a skeleton structure detecting every small curvature in the image. The skeleton looks like a network of blood vessels. Where Kinect skeleton model finds the single connecting line through the body joints detecting mid position of silhouette contour. That's why in Kinect skeleton model it is easy to find the different joint angles when subject moves where in morphological skeleton model, it creates a unique signatures of whole body in different times. As morphological skeleton creates unique signature patterns, so coding on skeleton can be applied to crate skeleton function. In table (2) Fskel is first time coded using an equation that authors derived (Kresch and Malah,1998) and then again they applied three coding scheme on Fskel function to create $\mathrm{F}_{\text {Fskel }}(\mathrm{x})$. The difference between Fskel and $\mathrm{F}_{\mathrm{Fskel}}(\mathrm{x})$ is the amplitude rate or bit rate of the skeleton. Analysing this amplitude rate, a subject can be identified by his gait. A Kinect skeleton pattern gives us the angle and other distinct features from a human gait which can be used to train any neural network. 
Table 2. Coding skeleton functions (Maragos and Schafer, 1986)

\begin{tabular}{|c|c|c|c|c|}
\hline \multirow[t]{2}{*}{ Image coded } & \multirow{2}{*}{$\begin{array}{c}\text { Block Huffman } \\
P=2 \times 4\end{array}$} & \multicolumn{2}{|c|}{ Run length-Huffman } & \multirow{2}{*}{$\begin{array}{c}\text { Elias } \\
m=3\end{array}$} \\
\hline & & Common & Separate & \\
\hline Fskel & 625 & 479 & 420 & 344 \\
\hline$F_{\text {Fskel }}(x)$ & 779 & 633 & 574 & 498 \\
\hline
\end{tabular}

Table 3. Performance (F-SCORE in \%) comparison for 5 subjects (Sinha et al., 2013)

\begin{tabular}{|c|l|l|l|}
\hline $\begin{array}{c}\text { K-Means } \\
\text { with } \boldsymbol{x} \text { feature }\end{array}$ & ANN with $\boldsymbol{x}$ feature & ANN with y features & ANN with both $\boldsymbol{x}, \boldsymbol{y}$ feature \\
\hline 25.2 & 34 & 69 & 86 \\
\hline
\end{tabular}

In table 4 a Fuzzy rule is defined. This definition explains the common angle range and time required for different gait phase at different body joint position. To determine the phases of gait for particular gait cycle this Fuzzy definition gives an approximate termination points. From this the angular motion $\theta$ can be identified. Table 5 shows step length error of a patient who walked in robotic walker (Lim et al, 2015b). Experiment done on one subject with five trial. Using particle filter gives better result than not using it. The noise due to floor segmentation is reason of poor result of not using particle filter. This experiment was basically for rehabilitation purpose for patients.

\section{CONCLUSION}

In this paper, we have discussed gait analysis and recognition for forensic applications. Detail discussion on nature of available databases presented here. Understanding gait recognition methodologies and their applications discussed in broad level. Throughout the survey, we found that gait analysis has high impact in biomedical engineering, medical areas and forensic experiments. There is large scope in research in gait recognition. Gait recognition is so popular because it recognize people from distance. Silhouette Extraction methods are discussed

Table 4. Fuzzy rule definition (MacDonald et al., 2007)

\begin{tabular}{|l|l|l|l|l|}
\hline \multicolumn{1}{|c|}{ Hip angle } & \multicolumn{1}{c|}{ Knee angle } & \multicolumn{1}{c|}{ Ankle angle } & \multicolumn{1}{c|}{ Time } & \multicolumn{1}{c|}{ Phase } \\
\hline L & S & Not L & S & LR \\
\hline Not S & S & L & S & MST \\
\hline M & S & M & S & MST \\
\hline S & S & L & M & TST \\
\hline S & Not S & Don't Care & M & PSW \\
\hline M & Not S & S & M & ISW \\
\hline L & L & M & L & MSW \\
\hline L & S & M & L & TSW \\
\hline
\end{tabular}


Table 5. Mean and standard deviation of step length error (Lim et al., 2015b)

\begin{tabular}{|l|l|l|l|l|}
\hline \multirow{2}{*}{} & \multicolumn{2}{|c|}{ Without particle filter } & \multicolumn{2}{c|}{ With particle filter } \\
\cline { 2 - 5 } & \multicolumn{1}{|c|}{ Mean(cm) } & \multicolumn{1}{c|}{ STD(cm) } & \multicolumn{1}{c|}{ Mean $(\mathbf{c m})$} & STD(cm) \\
\hline 1 & 7.5 & 6.51 & 2.84 & 2.11 \\
\hline 2 & 6.61 & 7.02 & 1.96 & .71 \\
\hline 3 & 4.38 & 2.77 & 1.71 & 1.66 \\
\hline 4 & 5.3 & 4.68 & 2.16 & 0.87 \\
\hline 5 & 6.38 & 3.61 & 2.95 & 1.25 \\
\hline Mean & 6.03 & & 2.33 & \\
\hline
\end{tabular}

elaborately. Strong feature extraction methods are discussed. When people walk, there lower limb oscillate in simple harmonic motion which creates people's unique movement pattern (Yoo et.al, 2008). For this reason, Gait recognition is a highly important feature in surveillance. Overall, we have done a survey of recent gait researches.

\section{ACKNOWLEDGMENT}

This project is partially supported by the CMATER research laboratory of the Computer Science and Engineering Department, Jadavpur University, India, PURSE-II and UPEII project, Research Award (F.30-31/2016(SA-II)) from UGC, Government of India. 


\section{REFERENCES}

Ahn, J.-H., \& Byun, H. (2007). Human Silhouette Extraction Method Using Region Based Background Subtraction. Computer VisionComputer Graphics Collaboration Techniques, 4418, 412-420.

Ali, H., Dargham, J., Ali, C., \& Moung, E. (2011). Gait Recognition using Gait Energy Image. Sersc.Org, 4(3).

Arai, K., \& Asmara, R. (2012). Gait Recognition Method Based on Wavelet Transformation and Its Evaluation With Chinese Academy of Sciences (CASIA) Gait Database as A Human Gait Recognition Dataset. Ninth International Conference on Information Technology - New Generation, 656-661.

Arai, K., \& Asmara, R. (2013). 3D Skeleton model derived from Kinect Depth Sensor Camera and its application to walking style quality evaluations. International Journal (Toronto, Ont.), 2(7), 24-28.

Ball, A., Rye, D., Ramos, F., \& Velonaki, M. (2012). Unsupervised clustering of people from 'skeleton' data. Proceedings of the seventh annual ACM/IEEE international conference on Human-Robot Interaction - HRI '12, 225.

Bashir, K., Xiang, T., \& Gong, S. (2008). Feature selection on gait energy image for human identification. ICASSP, IEEE International Conference on Acoustics, Speech and Signal Processing - Proceedings, 985-988.

Bharadwaj \& Chanda. (2020). Person Re-identification by analyzing Dynamic Variations in Gait Sequences. ETCCS 2020, Springer LNEE Proceedings.

Boyd, J. E., \& Little, J. J. (2005). Biometrie gait recognition. Lecture Notes in Computer Science, 3161, 19-42.

Carbonaro, N., Lorussi, F., Tognetti, A., \& De Rossi, D. (2015). Evaluation of wearable KPF goniometers in knee flexion-extension measurement for daily-life applications. Proceedings of the 5th EAI International Conference on Wireless Mobile Communication and Healthcare - Transforming healthcare through innovations in mobile and wireless technologies.

Chang, I.-C., \& Lin, S.-Y. (2010). 3D human motion tracking based on a progressive particle filter. Pattern Recognition, 43(10), 3621-3635.

Chao, H., He, Y., Zhang, J., \& Feng, J. (2019). GaitSet: Regarding Gait as a Set for Cross-View Gait Recognition. Proceedings of the AAAI Conference on Artificial Intelligence, 33(16), 8126-8133.

Cheng, M. H., Ho, M. F., \& Huang, C. L. (2008). Gait analysis for human identification through manifold learning and HMM. Pattern Recognition, 41(8), 2541-2553.

Cho, N.-G., Yuille, A. L., \& Lee, S.-W. (2013). Adaptive occlusion state estimation for human pose tracking under self-occlusions. Pattern Recognition, 46(3), 649-661.

Cuntoor, N., Kale, A., \& Chellappa, R. (2003). Combining multiple evidences for gait recognition. 2003 IEEE International Conference on Acoustics, Speech, and Signal Processing, 2003. Proceedings. (ICASSP '03), 3, 33-6.

Derin Babacan, S., \& Thrasyvoulos, N. (2007). Pappas. Spatiotemporal Algorithm for Background Subtraction.

Duc, C., Salvia, P., Lubansu, A., Feipel, V., \& Aminian, K. (2014). A wearable inertial system to assess the cervical spine mobility: Comparison with an optoelectronic-based motion capture evaluation. Medical Engineering \& Physics, 36(1), 49-56.

Firmenichy, D., Brown, M., \& Süsstrunk, S. (2011). Multispectral interest points for RGB-NIR image registration. Proceedings - International Conference on Image Processing, ICIP, 181-184.

Fritz, S., \& Lusardi, M. (2009). Walking speed: The sixth vital sign. Journal of Geriatric Physical Therapy, $32(2), 1-5$.

Gafurov, D., \& Snekkenes, E. (2009). Gait recognition using wearable motion recording sensors. EURASIP Journal on Advances in Signal Processing.

Hikal. (2013). A Comparative Evaluation Study of Automated Gait Recognition based on Spatiotemporal Feature and Different Neural Network Classifiers. Academic Press.

Jan, T., \& Wang, W. (2012). Blind reverberation time estimation based on Laplace distribution. European Signal Processing Conference, 2050-2054. 
Juana, E., Santoyo-morales, J. E., \& Hasimoto-beltran, R. (2014). Redalyc. Video Background Subtraction in Complex Environments., 12, 527-537.

Kaewtrakulpong, P., \& Bowden, R. (2001). An Improved Adaptive Background Mixture Model for Real- time Tracking with Shadow Detection. Advanced Video Based Surveillance Systems.

Kim, H., Sakamoto, R., Kitahara, I., Toriyama, T., \& Kogure, K. (2007). Robust silhouette extraction technique using background subtraction.

Kolaric, D., Lipic, T., Grubisic, I., Gjenero, L., \& Skala, K. (2011). Application of infrared thermal imaging in blade system temperature monitoring. Proceedings ELMAR, 2011(September), 309-312.

Kondragunta, J., Wiede, C., \& Hirtz, G. (2019). Gait analysis for early Parkinson's disease detection based on deep learning. Current Directions in Biomedical Engineering, 5(1), 9-12. doi:10.1515/cdbme-2019-0003

Kresch, R., \& Malah, D. (1998). Skeleton-based morphological coding of binary images. IEEE Transactions on Image Processing, 7(10), 1387-1399.

Li, S. Z. (2006). A Near-infrared Image Based Face Recognition System. 7th International Conference on Automatic Face and Gesture Recognition (FGR06), 455-460.

Lim, C. D., Cheng, C., Wang, C., Chao, Y., \& Fu, L. (2015b). Depth Image Based Gait Tracking and Analysis via Robotic Walker. IEEE International Conference on Robotics and Automation (ICRA), 5916-5921.

Lim, C. D., Wang, C. M., Cheng, C. Y., Chao, Y., Tseng, S. H., \& Fu, L. C. (2015a). Sensory Cues Guided Rehabilitation Robotic Walker Realized by Depth Image-Based Gait Analysis. IEEE Transactions on Automation Science and Engineering, 13(1), 171-180. doi:10.1109/TASE.2015.2494067 PMID:26279642

Livne, M., Sigal, L., Troje, N. F., \& Fleet, D. J. (2012). Human attributes from 3D pose tracking. Computer Vision and Image Understanding, 116(5), 648-660.

López-Fernández, D., Madrid-Cuevas, F. J., Carmona-Poyato, Á., Marín-Jiménez, M. J., \& Muñoz-Salinas, R. (2014). The AVA Multi-View Dataset for Gait Recognition BT - Activity Monitoring by Multiple Distributed Sensing. Second International Workshop, AMMDS 2014.

Lu, J., \& Zhang, E. (2007). Gait recognition for human identification based on ICA and fuzzy SVM through multiple views fusion. Pattern Recognition Letters, 28(16), 2401-2411.

Lu, X. M., Eun, S. J., \& Whangbo, T. K. (2007). Vector silhouette extraction for generating blueprint. Proceedings of the IEEE International Conference on Automation and Logistics, ICAL 2007, 2946-2951.

Lun, R., \& Zhao, W. (2015). A Survey of Applications and Human Motion Recognition with Microsoft Kinect. Academic Press.

MacDonald, C., Smith, D., Brower, R., Ceberio, M., \& Sarkodie-Gyan, T. (2007). Determination of Human Gait Phase Using Fuzzy Inference. 10th IEEE International Conference on Rehabilitation Robotics, 661-665.

Mallikarjuna, B., Viswanathan, R, \& Naib, B. (2020). Feedback-based gait identification using deep neural network classification. Advance online publication. doi:10.31838/jcr.07.04.125

Maragos, P. A., \& Schafer, R. W. (1986). Morphological Skeleton Representation and Coding of Binary Images. IEEE Transactions on Acoustics, Speech, and Signal Processing, 34(5), 1228-1244.

Margiotta, N., Avitabile, G., \& Coviello, G. (2016). A wearable wireless system for gait analysis for early diagnosis of Alzheimer and Parkinson disease. 2016 5th International Conference on Electronic Devices, Systems and Applications (ICEDSA), 1-4.

Martek, C. (2010). A Survey of Silhouette-Based Gait Recognition Methods. Academic Press.

Muramatsu, D., Makihara, Y., Iwama, H., Tanoue, T., \& Yagi, Y. (2013). Gait verification system for supporting criminal investigation. Proceedings - 2nd IAPR Asian Conference on Pattern Recognition, ACPR 2013, 747-748.

Panda, D. K., \& Meher, S. (2013). A Gaussian mixture model with Gaussian weight learning rate and foreground detection using neighbourhood correlation. Asia Pacific Conference on Postgraduate Research in Microelectronics and Electronics, 158-163. 
Papi, Bo, \& McGregor. (2018). A flexible wearable sensor for knee flexion assessment during gait. Gait \& Posture, 62, 480-483.

Papi, E., Koh, W. S., \& McGregor, A. H. (2017). Wearable technology for spine movement assessment: A systematic review. Journal of Biomechanics, 64, 186-197.

Parikesit, E., Mengko, T. L. R., \& Zakaria, H. (2011). Wearable gait measurement system based on accelerometer and pressure sensor. Proceedings - International Conference on Instrumentation, Communication, Information Technology and Biomedical Engineering 2011, ICICI-BME 2011, 395-398.

Pushpa \& Arumugam. (2010). An Efficient Gait Recognition System For Human Identification Using Modified ICA. International Journal of Computer Science \& Information Technology. 2.

Putzhammer, A., Perfahl, M., Pfeiff, L., \& Hajak, G. (2005). Gait disturbances in patients with schizophrenia and adaptation to treadmill walking. Psychiatry and Clinical Neurosciences, 59(3), 303-310.

Qi, Y., Soh, C. B., Gunawan, E., Low, K.-S., \& Thomas, R. (2016). Assessment of Foot Trajectory for Human Gait Phase Detection Using Wireless Ultrasonic Sensor Network. IEEE Transactions on Neural Systems and Rehabilitation Engineering, 24(1), 88-97.

Rampp, A., Barth, J., Schülein, S., Gaßmann, K. G., Klucken, J., \& Eskofier, B. M. (2015). Inertial Sensor-Based Stride Parameter Calculation From Gait Sequences in Geriatric Patients. IEEE Transactions on Biomedical Engineering, 62(4), 1089-1097.

Rani, M., \& Arumugam, G. (2010). An Efficient Gait Recognition System For Human Identification Using Modified ICA. International Journal of Computer Science and Information Technology, 2.

Razeghi, M., Lim, H., Tsao, S., Seo, H., \& Zhang, W. (2007). Thermal imaging based on high-performance InAs/InP quantum-dot infrared photodetector operating at high temperature. Conference Proceedings - Lasers and Electro-Optics Society Annual Meeting-LEOS, 2, 15-16.

Rida, I., Almaadeed, S., \& Bouridane, A. (2015). Improved gait recognition based on gait energy images. Proceedings of the International Conference on Microelectronics, ICM, 40-43.

Rodriguez-Gomez, R., Fernandez-Sanchez, E. J., Diaz, J., \& Ros, E. (2012). FPGA implementation for real-time background subtraction based on horprasert model. Sensors (Basel), 12(1), 585-611.

Sanders \& Gillig. (2010). Gait and its assessment in psychiatry. Psychiatry, 7(7), 38-43.

Sayeed, T., Sama, A., Catala, A., \& Cabestany, J. (2013). Comparison and adaptation of step length and gait speed estimators from single belt worn accelerometer positioned on lateral side of the body. 2013 IEEE 8th International Symposium on Intelligent Signal Processing, WISP 2013 - Proceedings, 14-20.

Schiel, J., \& Green, R. (2013). Adaptive human silhouette extraction with chromatic distortion and contour tracking. International Conference Image and Vision Computing New Zealand, 288-292.

Seely. (2010, July). On a three-dimensional gait recognition system by. Analysis.

Shutler, J., Grant, M., Nixon, M. S., \& Carter, J. N. (2002). On a Large Sequence-Based Human Gait Database. Proceedings Fourth International Conference Recent Advances in Soft Computing, 66-72.

Sinha, A., Chakravarty, K., \& Bhowmick, B. (2013). Person Identification using Skeleton Information from Kinect. The Sixth International Conference on Advances in Computer-Human Interactions (ACHI), 101-108.

Sivapalan, S., Chen, D., Denman, S., Sridharan, S., \& Fookes, C. (2011). Gait energy volumes and frontal gait recognition using depth images. 2011 International Joint Conference on Biometrics, IJCB.

Sokolova, A., \& Konushin, A. (2019). Human identification by gait from event-based camera. Proceedings of the 16th International Conference on Machine Vision Applications, MVA 2019, 1-6.

Sun, C., Wang, C., \& Lai, W. (2019). Gait analysis and recognition prediction of the human skeleton based on migration learning. Physica A, 532, 121812.

Teixeira, L. F., Cardoso, J. S., \& Corte-Real, L. (2007). Object segmentation using background modelling and cascaded change detection. Journal of Multimedia, 2(5), 55-65. 
Van Mastrigt, N. M., Celie, K., Mieremet, A. L., Ruifrok, A., \& Geradts, Z. (2018). Critical review of the use and scientific basis of forensic gait analysis. Forensic Sciences Research, 3(3), 183-193. $10.1080 / 20961790.2018 .1503579$

Wang, L., Tan, T., Ning, H., \& Hu, W. (2003). Silhouette analysis-based gait recognition for human identification. Pattern Analysis and Machine Intelligence. IEEE Transactions on, 25(12), 1505-1518.

Wang, Y. (2019). EV-Gait : Event-based Robust Gait Recognition using Dynamic Vision Sensors. Proceedings of IEEE Conference on Computer Vision and Pattern Recognition (CVPR), 6358-6367.

Wen, L., Qian, J., Hu, X., Shen, L., Wu, X., \& Yu, C. (2013). Gait measurement and quantitative analysis in patients with Parkinson's disease for rehabilitation assessment. 2013 IEEE International Conference on Robotics and Biomimetics, ROBIO 2013, 286-291.

Wen, S., Wang, F., Wu, C., \& Zhang, Y. (2010). Gait data de-noising based on improved EMD. Chinese Control and Decision Conference, CCDC 2010, 2766-2770.

Yang, C.-C., Hsu, Y.-L., Shih, K.-S., \& Lu, J.-M. (2011). Real-Time Gait Cycle Parameter Recognition Using a Wearable Accelerometry System. Sensors (Basel), 11(12), 7314-7326.

Yang, X., Zhang, T., Zhou, Y., \& Yang, J. (2008). Gabor phase embedding of gait energy image for identity recognition. Proceedings - 2008 IEEE 8th International Conference on Computer and Information Technology, CIT 2008, 361-366.

Yang, Y., Tu, D., \& Li, G. (2014). Gait recognition using flow histogram energy image. Proceedings - International Conference on Pattern Recognition, 444-449.

Yoo, J., Hwang, D., Moon, K., \& Nixon, M. S. (2008). Automated Human Recognition by Gait using Neural Network. First Workshops on Image Processing Theory, Tools and Applications, Sousse, Tunisia, 2008, 1-6. doi:10.1109/IPTA.2008.4743792

Yu, S., Tan, D., \& Tan, T. (2006). A framework for evaluating the effect of view angle, clothing and carrying condition on gait recognition. Proceedings - International Conference on Pattern Recognition, 4, 441-444.

Zhao, X., \& Du, Y. (2010). Research on gait-based human identification. ICCASM 2010 - 2010 International Conference on Computer Application and System Modeling, Proceedings, 12, 480-482.

Zheng, S., Huang, K., Tan, T., \& Tao, D. (2012). A cascade fusion scheme for gait and cumulative foot pressure image recognition. Pattern Recognition, 45(10), 3603-3610.

Zhuang, Y., \& Chen, C. (2007). Efficient silhouette extraction with dynamic viewpoint. Proceedings of the IEEE International Conference on Computer Vision.

Shantanu Jana received his B. Tech degree in CSE from MCKV Institute of Engineering. He received his M.Tech degree in Computer Technology from Jadavpur University. He was Junior UPE Fellow under the UGC funded "University with Potential for Excellence- Phase-II" Scheme of Jadavpur University.

Nibaran Das received his B.Tech degree in Computer Science and Technology from Kalyani Govt. Engineering College under Kalyani University, in 2003. He received his M.C.S.E and Ph. D.(Engg.) degree from Jadavpur University, in 2005 and 2012 respectively. He joined Jadavpur University as a faculty member in 2006. His areas of current research interest are OCR of handwritten text, optimization techniques, Deep Learning, and image processing. He has been an editor of Bengali monthly magazine "Computer Jagat" since 2005.

Subhadip Basu Subhadip Basu received his Ph.D. (Engineering) degree from Jadavpur University in 2006 and joined the Computer Science and Engineering Department of Jadavpur University in the same year, where he is currently working as an Associate Professor. He is the recipient of the Research Award from UGC, BOYSCAST and FASTTRACK fellowships from DST, Govt. of India, HIVIP fellowship from Hitachi, Japan and EMMA fellowships from the European Union. He is a senior member of IEEE and life member of IUPRAI (Indian unit for IAPR).

Mita Nasipuri received her B.E. Tel. E., M.E. Tel. E., and Ph. D. (Engg.) degrees from Jadavpur University, in 1979, 1981 and 1990, respectively. Prof. Nasipuri has been a faculty member of J.U. since 1987. Her current research interest includes image processing, pattern recognition, and multimedia systems. She is a senior member of the IEEE, U.S.A., Fellow of I.E. (India) and W.B.A.S.T., Kolkata, India. 